\title{
Involvement of gut microbiota in the association between gastrointestinal motility and 5-HT expression/M2 macrophage abundance in the gastrointestinal tract
}

\author{
MO YANG $^{1,2}$, HIROKAZU FUKUI ${ }^{1}$, HIROTSUGU EDA ${ }^{1}$, YOSHITAKA KITAYAMA ${ }^{1}$, KEN HARA ${ }^{1}$, \\ MIO KODANI $^{1}$, TOSHIHIKO TOMITA ${ }^{1}$, TADAYUKI OSHIMA ${ }^{1}$, JIRO WATARI $^{1}$ and HIROTO MIWA ${ }^{1}$ \\ ${ }^{1}$ Division of Gastroenterology, Department of Internal Medicine, Hyogo College of Medicine, Nishinomiya, \\ Hyogo 663-8501, Japan; ${ }^{2}$ Department of Digestive Diseases, Tianjin Medical \\ University General Hospital, Heping, Tianjin 300052, P.R. China
}

Received May 22, 2016; Accepted February 24, 2017

DOI: $10.3892 / \mathrm{mmr} .2017 .6955$

\begin{abstract}
Serotonin (5-hydroxytryptamine; 5-HT) may be a key player in gastrointestinal (GI) motility and the GI immune system. In the present study, the effect of gut microbiota on the association between GI motility, and 5-HT expression and macrophage abundance in the GI tract was examined. Germ-free (GF) mice (6 weeks old) were orally administered a fecal bacterial suspension prepared from specific pathogen-free mice and their GI tissues were evaluated 4 weeks later. The expression of 5-HT and mannose receptor (MR) was examined by immunohistochemistry, and GI transit time (GITT) was measured by administration of carmine red solution. The numbers of 5-HT-positive endocrine cells and muscularis MR-positive macrophages were significantly increased in the upper GI and colon of GF mice subjected to fecal transplantation (FT) compared with control GF mice without FT. GITT was significantly decreased in GF mice subjected to FT compared with GF mice without FT, and negatively correlated with the numbers of 5-HT-positive cells in the upper GI and muscularis MR-positive macrophages throughout the GI tract. The numbers of 5-HT-positive endocrine cells and muscularis MR-positive macrophages were significantly correlated throughout the GI tract. The present results suggest that the gut microbiota is involved in the association between accelerated GI motility and induction of the 5-HT/muscularis MR-positive macrophage axis in the GI tract.
\end{abstract}

Correspondence to: Dr Hirokazu Fukui, Division of Gastroenterology, Department of Internal Medicine, Hyogo College of Medicine, 1-1 Mukogawa, Nishinomiya, Hyogo 663-8501, Japan E-mail: hfukui@hyo-med.ac.jp

Abbreviations: GI, gastrointestinal; 5-HT, 5-hydroxytryptamine; GF, germ-free; SPF, specific pathogen-free; MR, mannose receptor; FT, fecal transplantation; GITT, gastrointestinal transit time

Key words: 5-HT, M2 macrophage, microbiota, motility, GI tract

\section{Introduction}

It is widely accepted that microbiota in the gut serves a pivotal role in host physiology by interacting with the immune and neuroendocrine systems in gastrointestinal (GI) tissues (1-4). In addition, gut hormones, which are key players in the neuroendocrine system, have various important functions in secretion, metabolism and motility $(5,6)$. Therefore, it is hypothesized that gut microbiota may be involved in the fine-tuning of GI motility through modification of neuroendocrine signaling; however, this hypothesis has not been completely tested to date.

Serotonin or 5-hydroxytryptamine (5-HT), predominantly synthesized by GI enterochromaffin cells (7), acts as a neurotransmitter and/or local hormone in the enteric nervous system, resulting in alteration of GI motility $(7,8)$. Gut microbiota is known to affect the behavior of 5-HT-producing enterochromaffin cells in the GI mucosa (9), and have been reported to regulate GI motility by modifying 5-HT expression in the GI tract (10). Previously, evidence that 5-HT signaling affects the enteric nervous system and the immune system in the GI wall has been reported (8). 5-HT is important in M2 macrophage polarization and cytokine production in GI tissues (11). In addition, it has been reported that macrophages in the muscular layer may act on the enteric nervous system via the GI wall (2), although the mechanism underlying the 5-HT/muscularis macrophage axis and its effect on GI motility remains unclear. 5-HT has been extensively studied as a therapeutic target for functional GI disorders, which involve a significant disturbance in GI motility (7). In this regard, it would be meaningful to clarify the role of the 5-HT/muscularis macrophage axis in GI motility in humans. However, GI muscular layer tissues cannot be collected from humans, therefore animal experiments are required to approach these issues. In addition, it would be of interest to determine how gut microbiota are involved in the 5-HT/muscularis macrophage axis in GI motility in humans. However, this theme is also difficult to examine in the human body, and therefore germ-free (GF) animals are vital to examine the effect of gut microbiota on GI physiology. In the present study, intestinal 
microorganisms were transplanted into GF mice in order to examine how gut microbiota affects the association between GI motility, and 5-HT expression and M2 macrophage abundance in the GI tract.

\section{Materials and methods}

Animal and experimental design. A total of 22 GF (6 or 10 weeks old; male; ICR strain; weight, 26 to $40 \mathrm{~g}$ ) and 6 specific pathogen-free (SPF; 7 to 9 weeks old; male; ICR strain; weight, 33 to $42 \mathrm{~g}$ ) mice were purchased from CLEA Japan, Inc. (Tokyo, Japan). Fecal suspensions were freshly prepared from SPF mice by 10 -fold dilution of colonic content with saline, as reported previously (12). GF mice at 6 weeks of age were orally administered these fecal suspensions to reconstitute the intestinal flora. Thereafter, the GF mice that had undergone fecal transplantation (FT) were housed under SPF conditions and sacrificed 4 weeks following FT. Non-microflora-reconstituted GF mice at 6 and 10 weeks of age were used as controls. The experimental protocol was approved by the Animal Use and Care Committee at Hyogo College of Medicine (Nishinomiya, Japan).

Histopathological evaluation. The GI tissues were removed from experimental mice and fixed in $10 \%$ buffered formalin overnight at room temperature, sliced perpendicularly to the surface, embedded in paraffin, and cut into sections $4-\mu \mathrm{m}$ thick. The sections were deparaffinized in xylene, rehydrated in a series of ethanol and stained with $100 \%$ hematoxylin (room temperature for $2 \mathrm{~min}$ ) and $0.1 \%$ eosin (room temperature for $1 \mathrm{~min}$ ) for histopathological observation under a light microscope (Olympus CX41; Olympus Corporation, Tokyo, Japan).

Immunohistochemistry. Immunohistochemical staining for 5-HT and mannose receptor (MR; a marker of M2-polarized macrophages) was performed using the Envision Kit (Dako; Agilent Technologies, Inc., Santa Clara, CA, USA), according to the manufacturer's protocol. The primary antibodies used were: anti-5-HT (1:100,000 dilution; cat. no. IST-20080; ImmunoStar, Inc., Hudson, WI, USA) and anti-MR (1:1,000 dilution; cat. no. ab64693; Abcam, Cambridge, UK). As previously described (13), the sections were deparaffinized, rehydrated, placed in $1 \mathrm{X}$ Dako REAL Target Retrieval Solution (Dako; Agilent Technologies, Inc.), and heated in the microwave for $20 \mathrm{~min}$. The sections were subsequently preincubated with $0.3 \% \mathrm{H}_{2} \mathrm{O}_{2}$ in methanol for $25 \mathrm{~min}$ at room temperature to quench endogenous peroxidase activity. The sections were incubated with primary antibodies for $1 \mathrm{~h}$ at room temperature. The slides were incubated with horseradish peroxidase-conjugated secondary antibodies (ready-to-use; cat. nos. K4001 or K4003; Dako; Agilent Technologies, Inc.) at room temperature for $30 \mathrm{~min}$, visualized by 3,3'-diaminobenzide tetrahydrochloride with $0.05 \% \mathrm{H}_{2} \mathrm{O}_{2}$ for $3 \mathrm{~min}$, and counterstained with Mayer's hematoxylin. Under a light microscope (Olympus CX41; Olympus Corporation), 5-HT-positive epithelial cells were counted by eye in a $1,000 \mu \mathrm{m}$ stretch of the entire length of well-oriented tissue sections in at least 5 randomly selected fields from the stomach to colon of each mouse, and the average was calculated. Similarly, the number of MR-positive cells was evaluated in the lamina propria and muscle layer throughout the GI tract.

GI transit time (GITT). GITT was measured as described previously (14). The mice orally received $0.3 \mathrm{ml}$ of $0.5 \%$ methylcellulose solution including 6\% carmine red (Wako Pure Chemical Industries, Ltd., Osaka, Japan). Following administration of the solution, the mice were allowed free access to food and water ad libitum until the first red fecal pellet appeared. GITT was determined as the time period between oral gavage and the appearance of the first red fecal pellet.

Statistical analysis. Statistical analyses were performed using StatView 5.0 software (SAS Institute, Inc., Buckinghamshire, UK). All values were expressed as the mean \pm standard error of the mean. Differences between two animal groups were analyzed using the Mann-Whitney U-test. The correlation among GITT, 5-HT expression and MR expression was assessed by linear regression analysis. $\mathrm{P}<0.05$ was considered to indicate a statistically significant difference.

\section{Results}

Expression of 5-HT in the GI tract of FT-treated mice. In GF mice, 5-HT-positive cells were observed throughout the GI epithelium (Fig. 1). The 5-HT immunoreactivity was localized in the cytoplasm of ovoid or pyramidal epithelial cells throughout the GI mucosa, a staining pattern that was morphologically compatible with endocrine cells (Fig. 1). Expression of 5-HT protein was subsequently evaluated in the GI tissues of 6-week-old GF mice at 4 weeks post-FT, and age-matched control mice that did not receive FT (Figs. 1 and 2). The results demonstrated that the number of 5-HT-positive cells was significantly increased in the colonic mucosa (proximal and distal colon) of GF mice with FT, compared with mice without FT (Fig. 2). In addition, the number of 5-HT-positive cells was significantly increased in not only the gastric mucosa (stomach), but also the proximal small-intestinal mucosa (jejunum) in mice with FT compared with mice without FT (Fig. 2).

Expression of MR in the GI tract of FT-treated mice. MR protein expression, as a marker of M2-polarized macrophages, was evaluated in GI tissues by immunohistochemistry. In GF mice, immunoreactivity for MR was detected in immune cells of, not only the lamina propria (mucosal layer), but also the muscular layer throughout the GI tract (Fig. 3). Following FT, the number of MR-positive cells was significantly increased in the lamina propria of the stomach and colon in GF mice, compared with mice without FT (Fig. 4). The number of MR-positive cells was also significantly increased in the muscular layer of the upper GI tract (stomach and jejunum) and colon (proximal and distal) of FT-treated mice, compared with mice without FT (Fig. 4). Notably, MR-positive cells were frequently present around the myenteric neural cells between the circular and longitudinal muscle layers throughout the GI tract (Fig. 3). These results suggest that treatment with FT is associated with increased infiltration of MR-positive cells, presumably M2 macrophages, into both the mucosal and the muscular layers throughout the GI tract. 


\section{GF without FT}

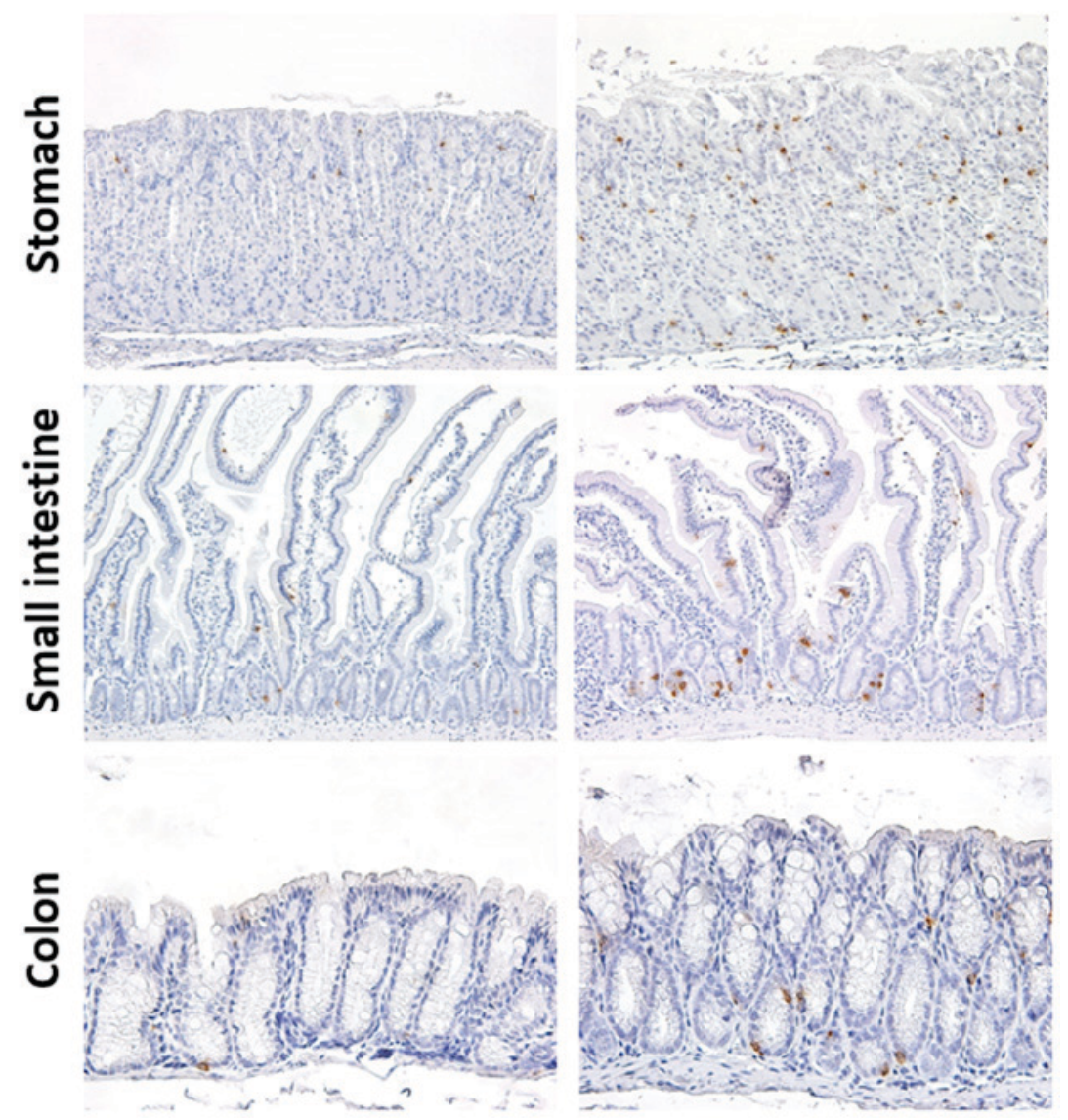

Figure 1. Expression of 5-HT in the gastrointestinal tract of GF mice without or with FT. 5-HT expression was examined by immunohistochemistry in sections from stomach, small intestine and colon tissues of GF mice treated with FT and of age-matched untreated mice (GF without FT). 5-HT is expressed in the cytoplasm of ovoid or pyramidal epithelial cells in the epithelium of stomach, small intestine and colon. 5-HT, 5-hydroxytryptamine; GF, germ free; FT, fecal transplantation.

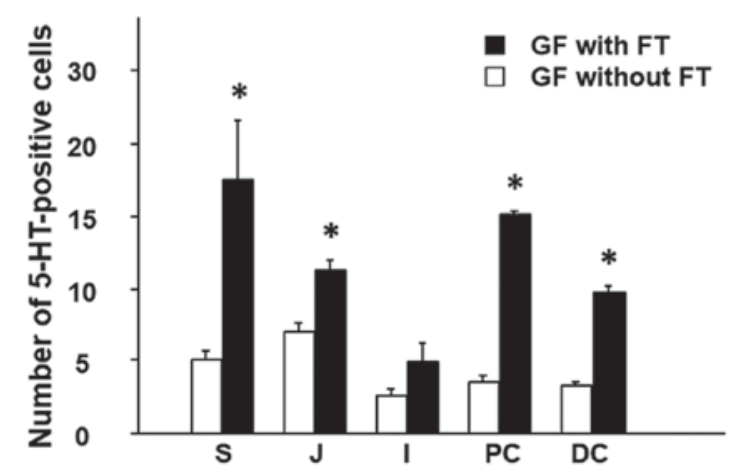

Figure 2. 5-HT-positive cells in the gastrointestinal tract of GF mice with or without fecal transplantation. Quantification of immunohistochemistry staining for 5-HT-positive cells in tissue sections from GF mice treated with FT and of age-matched untreated mice (GF without FT). Results are expressed as the mean \pm standard error of the mean of 3 samples per group. ${ }^{*} \mathrm{P}<0.05$ vs. GF without FT. 5-HT, 5-hydroxytryptamine; GF, germ free; FT, fecal transplantation; S, stomach; J, jejunum; I, ileum; PC, proximal colon; DC, distal colon.

GITT and its association with 5-HT or MR expression in GF mice. GITT was significantly decreased in GF mice subjected to FT compared with age-matched GF mice without FT (Fig. 5). The correlation between GITT and 5-HT or MR expression was investigated in the experimental mice (FT-treated and untreated) by linear regression analysis. GITT was negatively correlated with the number of 5-HT-positive cells in the gastric and small-intestinal mucosa (Fig. 6), whereas no correlation was evident for GITT with 5-HT-positive cells in the colonic mucosa (Fig. 6). GITT was negatively correlated with the number of MR-positive cells in the lamina propria and the number of MR-positive cells in the muscular layer throughout the GI wall (Fig. 7).

Association between 5-HT and MR expression in GI tissues of GF mice. The association between 5-HT and MR expression in the experimental mice (FT-treated and untreated) was also investigated by linear regression analysis. In the stomach and small intestine, the number of MR-positive cells in the mucosal layer was positively correlated with the number of 5-HT-positive cells, whereas no such correlation was evident in the colon (Fig. 8). The number of MR-positive cells in the muscular layer was positively correlated with the number of 5-HT-positive cells throughout the GI wall (Fig. 8).

\section{Discussion}

In the present study, 5-HT was demonstrated to be expressed in endocrine cells throughout the GI mucosa in GF mice, and 

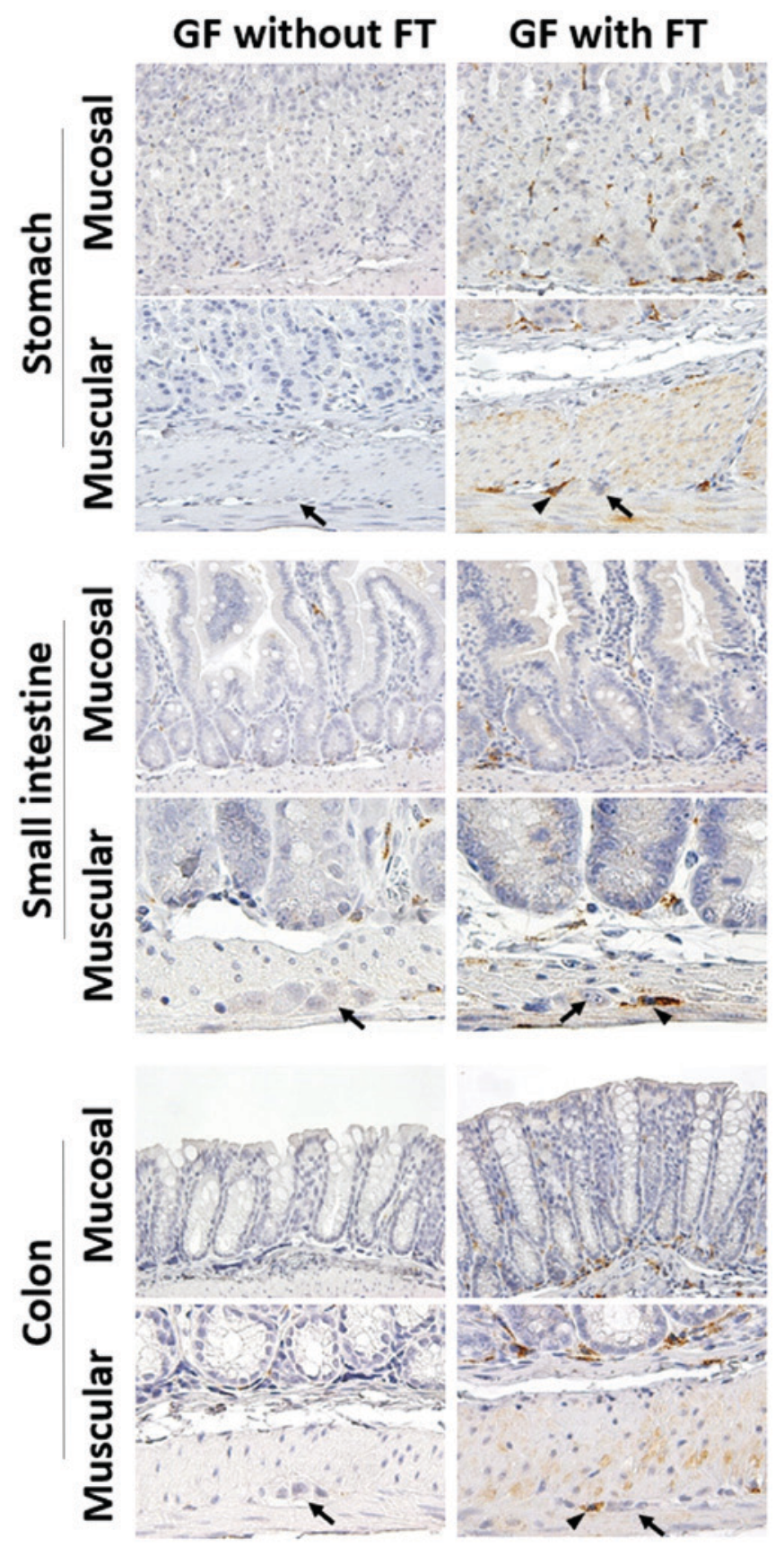

Figure 3. Expression of MR in the gastrointestinal tract of GF mice without or with FT. MR expression was examined by immunohistochemistry in sections from stomach, small intestine and colon tissues of GF mice treated with FT and of age-matched untreated mice (GF without FT). Arrows indicate neural cells and arrow heads indicate MR-positive cells in the muscular layer. MR, mannose receptor; GF, germ free; FT, fecal transplantation.

the number of 5-HT-positive cells was increased in GF mice following transplantation of gut microbiota, consistent with previous reports (10). Although in humans the population of 5-HT-positive endocrine cells is larger in the upper GI and rectum (15), no significant differences were observed among the various parts of the GI tract of the GF mice examined in the present study. However, the number of 5-HT-positive endocrine cells increased in the upper GI and colon of FT-treated GF mice compared with untreated mice, resulting in a distribution of 5-HT-positive cells in the GI tract that resembled that of humans. This finding suggests that gut microbiota
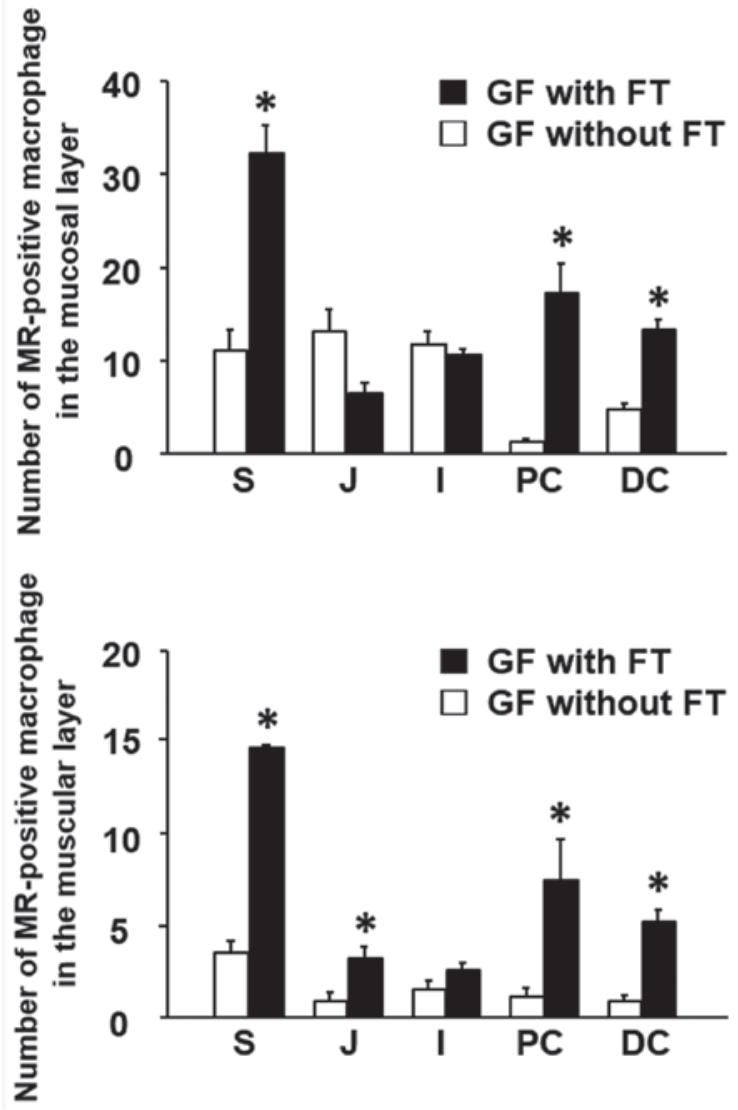

Figure 4. MR-positive cells in the gastrointestinal tract of GF mice with or without FT. Quantification of immunohistochemistry staining for MR-positive cells (counted separately for the mucosal and the muscular layers) of tissue sections from GF mice treated with FT and of age-matched untreated mice (GF without FT). Results are expressed as the mean \pm standard error of the mean of 3 samples per group. ${ }^{*} \mathrm{P}<0.05$ vs. GF without FT. MR, mannose receptor; GF, germ free; FT, fecal transplantation; S, stomach; J, jejunum; I, ileum; PC, proximal colon; DC, distal colon.

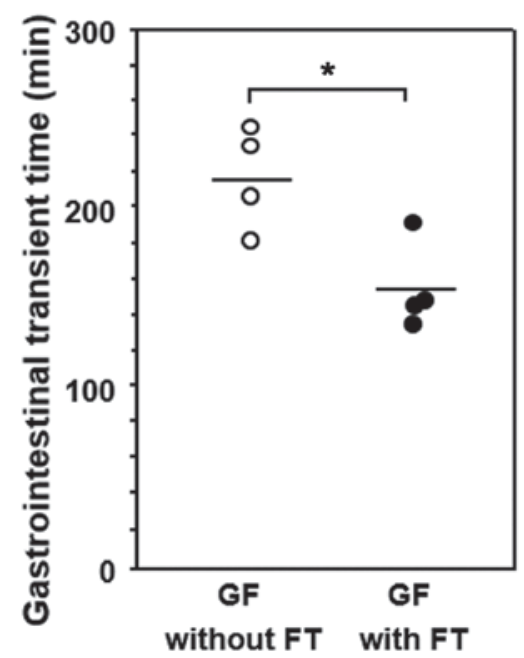

Figure 5. Gastrointestinal transit time in GF mice without or with FT. The mice in the two groups were age-matched (10 weeks old). Results are shown for 4 mice per group, with bars indicating mean values. "P<0.05 vs. GF without FT. GF, germ free; FT, fecal transplantation.

may be essential in determining the distribution profile of 5-HT-producing endocrine cells in the GI tract. 

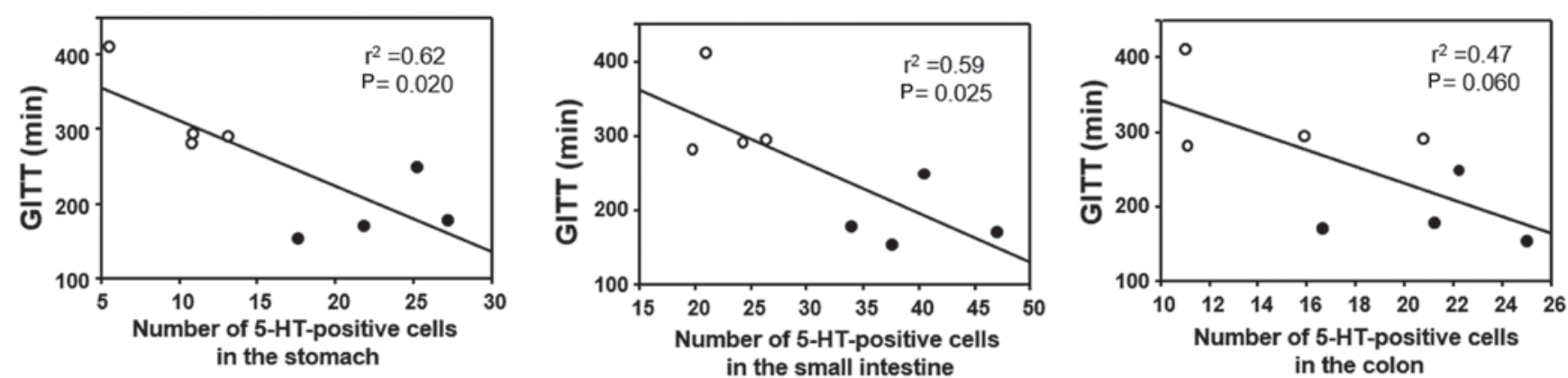

Figure 6. Correlation between GITT and 5-HT expression in the stomach, small intestine and colon of GF mice. Results from FT-treated GF mice are presented in black circles, whereas results from age-matched GF mice without FT are presented in white circles. P-values were obtained by linear regression analysis comparing GITT with the number of 5-HT-positive cells in the stomach, small intestine and colon, respectively. GITT, gastrointestinal transit time; GF, germ free; FT, fecal transplantation.
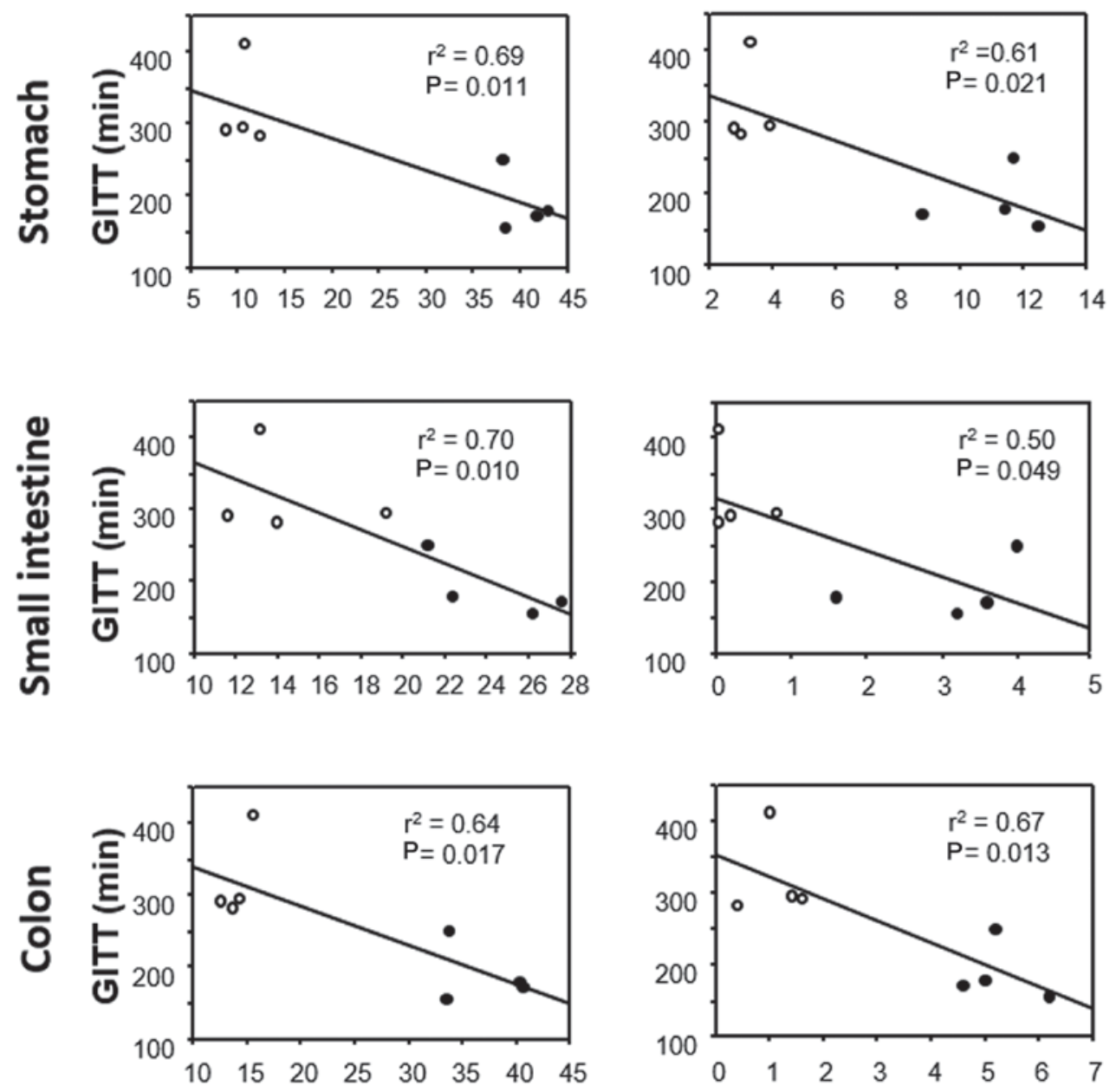

\section{Number of MR-positive macrophage in the mucosal layer}

\section{Number of MR-positive macrophage in the muscular layer}

Figure 7. Correlation between GITT and MR expression in the mucosal and muscular layers of the stomach, small intestine and colon of GF mice. Results from FT-treated GF mice are presented in black circles, whereas results from age-matched GF mice without FT are presented in white circles. P-values were obtained by linear regression analysis comparing GITT with the number of MR-positive macrophages in the mucosal/muscular layer of the stomach, small intestine and colon, respectively. GITT, gastrointestinal transit time; MR, mannose receptor; GF, germ free; FT, fecal transplantation.

The distribution of M2 macrophages was also investigated by immunostaining for MR (16), and the results revealed that MR-positive macrophages were present in the lamina propria and the muscular layer throughout the GI wall. Notably,
MR-positive macrophages were also distributed around myenteric neural cells between the circular and longitudinal muscle layers of the GI wall, suggesting that these macrophages may serve a role in the physiology of the enteric nervous system. The 

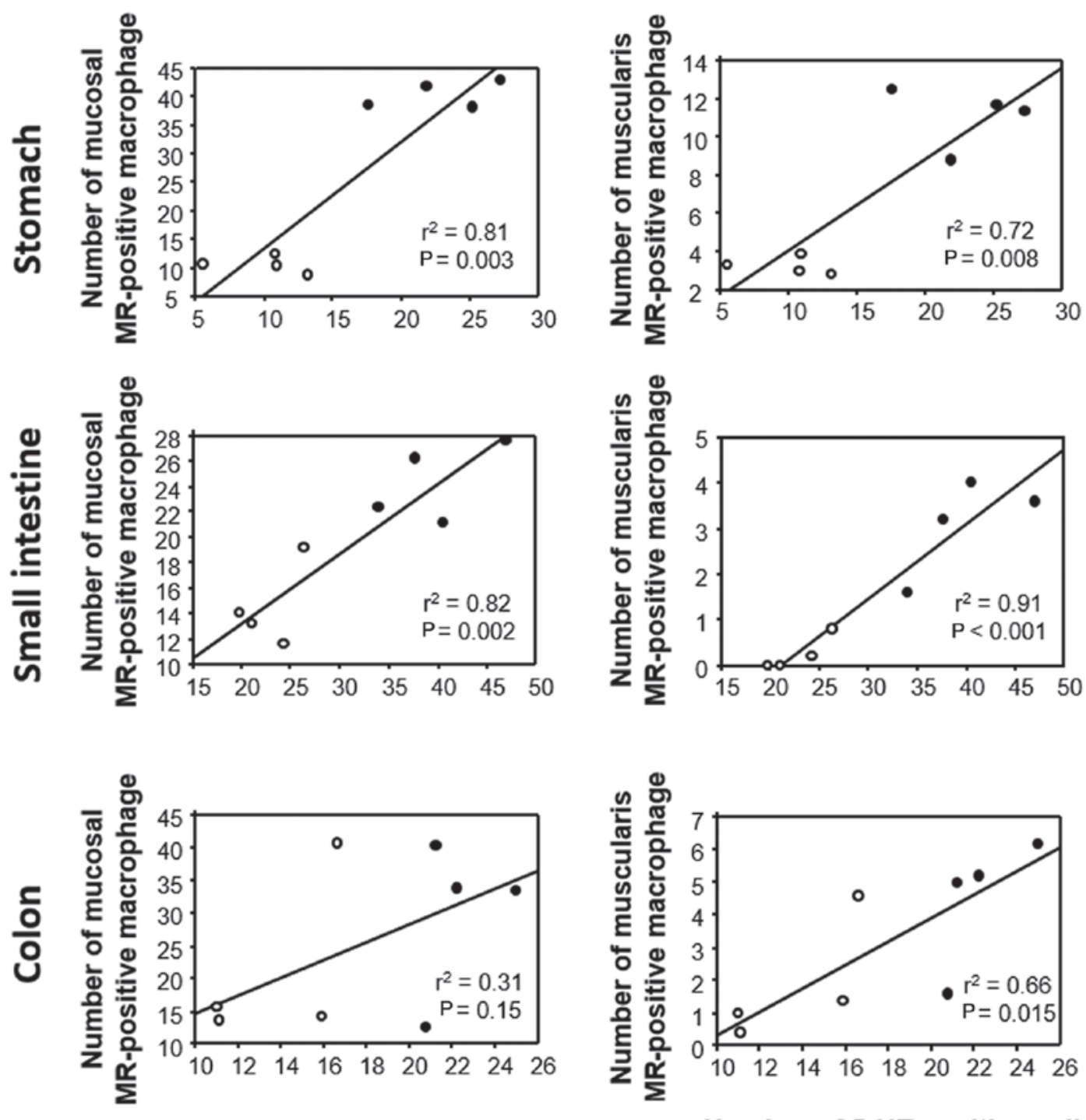

Number of 5-HT-positive cells

\section{Number of 5-HT-positive cells}

Figure 8. Correlation between 5-HT and mucosal/muscularis MR expression in the stomach, small intestine and colon of GF mice. Results from FT-treated GF mice are presented in black circles, whereas results from age-matched GF mice without FT are presented in white circles. P-values were obtained by linear regression analysis comparing the number of mucosal and muscularis MR-positive macrophages with the of number of 5-HT-positive cells in the stomach, small intestine and colon, respectively. 5-HT, 5-hydroxytryptamine; MR, mannose receptor; GF, germ free; FT, fecal transplantation.

numbers of M2 macrophages in the muscular layer were markedly increased in the upper GI tract and colon of FT-treated GF mice, suggesting that gut microbiota may promote infiltration of macrophages into the GI muscular layer.

Furthermore, the role of gut microbiota in GI motility was examined and demonstrated to be suppressed in control untreated GF mice in comparison with mice with gut microbiota, consistent with previous reports $(17,18)$. Conversely, this result may suggest that transplantation of gut microbiota accelerated GI motility in GF mice. To clarify the mechanism by which gut microbiota may have accelerated GI motility, the correlation between GITT and 5-HT expression or MR-positive macrophages in the GI tract was further examined. The results demonstrated that GITT was negatively correlated with the number of 5-HT-positive cells in the upper GI tract, suggesting that the increase of 5-HT expression is associated with acceleration of GI motility. A previous study has also reported that 5-HT promotes motility of the GI tract (19). In addition, GITT was negatively correlated with the number of MR-positive macrophages in the muscular layer of the GI tract. Recently, Muller et al (20) have reported that musclaris macrophages are important in GI motility through cross-talk with enteric nerve cells through bone morphogenetic protein 2 . In the present study, although the specific mediators produced by M2 macrophages were not identified, the numbers of M2 macrophages following FT were demonstrated to be significantly correlated with acceleration of GI motility.

Since the movement of the GI tract is orchestrated by the enteric nervous system $(21,22)$, the mechanism by which endocrine-produced 5-HT acts on the enteric nervous system is of great interest. It is believed that the 5-HT secreted by enterochromaffin cells acts locally on the submucosal and the myenteric neural cells in a paracrine manner $(7,23)$. In addition, as 5-HT may also act on immune cells that express its receptor (8), 5-HT-induced mediators from the immune cells may affect the enteric nervous system function (24). Notably, 
previous evidence suggests that 5-HT is important in the polarization of macrophages toward an M2 phenotype (11). In this context, it was notable that in the present study the number of MR-positive cells in the muscular layer was positively correlated with the number of 5-HT-positive cells throughout the GI wall. Therefore, it is speculated that 5-HT may act on the enteric nervous system directly or indirectly via macrophage stimulation.

In conclusion, the present study has demonstrated that the numbers of 5-HT-positive endocrine cells and muscularis MR-positive macrophages are positively correlated in the GI tract, and are significantly increased in the upper GI and colon of GF mice following transplantation of gut microbiota, compared with age-matched untreated mice. Furthermore, GITT was decreased in GF mice following FT compared with untreated mice, and it was negatively correlated with the number of 5-HT-positive endocrine cells and muscularis MR-positive macrophages. The precise mechanism by which gut microbiota accelerated GI motility remains unknown. However, the present data suggest that gut microbiota may be important in inducing increased numbers of 5-HT-producing endocrine cells and muscularis M2 macrophages in the GI tract, which may act on the enteric nervous system to modulate GI function.

\section{Acknowledgements}

The present work was supported in part by Grants-in-aid for Scientific Research (grant no. 26460953) from the Japanese Ministry of Education, Culture, Sports, Science and Technology. The authors of the present study would like to thank Miss Mayumi Yamada and Miss Chiyomi Itoh (Hyogo College of Medicine, Nishinomiya, Japan) for their technical assistance.

\section{References}

1. Furness JB, Rivera LR, Cho HJ, Bravo DM and Callaghan B: The gut as a sensory organ. Nat Rev Gastroenterol Hepatol 10: 729-740, 2013

2. Kabouridis PS and Pachnis V: Emerging roles of gut microbiota and the immune system in the development of the enteric nervous system. J Clin Invest 125: 956-964, 2015.

3. Psichas A, Reimann F and Gribble FM: Gut chemosensing mechanisms. J Clin Invest 125: 908-917, 2015.

4. Stasi C, Rosselli M, Bellini M, Laffi G and Milani S: Altered neuro-endocrine-immune pathways in the irritable bowel syndrome: The top-down and the bottom-up model. J Gastroenterol 47: 1177-1185, 2012.

5. Drucker DJ: The role of gut hormones in glucose homeostasis. J Clin Invest 117: 24-32, 2007.

6. Murphy KG and Bloom SR: Gut hormones and the regulation of energy homeostasis. Nature 444: 854-859, 2006.
7. Gershon MD and Tack J: The serotonin signaling system: From basic understanding to drug development for functional GI disorders. Gastroenterology 132: 397-414, 2007.

8. Baganz NL and Blakely RD: A dialogue between the immune system and brain, spoken in the language of serotonin. ACS Chem Neurosci 4: 48-63, 2013.

9. Uribe A, Alam M, Johansson O, Midtvedt $\mathrm{T}$ and Theodorsson E: Microflora modulates endocrine cells in the gastrointestinal mucosa of the rat. Gastroenterology 107: 1259-1269, 1994

10. Yano JM, Yu K, Donaldson GP, Shastri GG, Ann P, Ma L, Nagler CR, Ismagilov RF, Mazmanian SK and Hsiao EY: Indigenous bacteria from the gut microbiota regulate host serotonin biosynthesis. Cell 161: 264-276, 2015.

11. de las Casas-Engel M, Domínguez-Soto A, Sierra-Filardi E, Bragado R, Nieto C, Puig-Kroger A, Samaniego R, Loza M, Corcuera MT, Gómez-Aguado F, et al: Serotonin skews human macrophage polarization through HTR2B and HTR7. J Immunol 190: 2301-2310, 2013

12. Ogawa H, Fukushima K, Sasaki I and Matsuno S: Identification of genes involved in mucosal defense and inflammation associated with normal enteric bacteria. Am J Physiol Gastrointestinal Liver Physiol 279: G492-G499, 2000.

13. Ogata H, Sekikawa A, Yamagishi H, Ichikawa K, Tomita S, Imura J, Ito Y, Fujita M, Tsubaki M, Kato H, et al: GROa promotes invasion of colorectal cancer cells. Oncol Rep 24: 1479-1486, 2010.

14. Welch MG, Margolis KG, Li Z and Gershon MD: Oxytocin regulates gastrointestinal motility, inflammation, macromolecular permeability, and mucosal maintenance in mice. Am J Physiol Gastrointest Liver Physiol 307: G848-G862, 2014.

15. Sjölund K, Sandén G, Håkanson R and Sundler F: Endocrine cells in human intestine: An immunocytochemical study. Gastroenterology 85: 1120-1130, 1983.

16. Nakanishi Y, Nakatsuji M, Seno H, Ishizu S, Akitake-Kawano R, Kanda K, Ueo T, Komekado H, Kawada M, Minami M and Chiba T: COX-2 inhibition alters the phenotype of tumor-associated macrophages from M2 to M1 in ApcMin/+ mouse polyps. Carcinogenesis 32: 1333-1339, 2011.

17. Kashyap PC, Marcobal A, Ursell LK, Larauche M, Duboc H, Earle KA, Sonnenburg ED, Ferreyra JA, Higginbottom SK, Million M, et al: Complex interactions among diet, gastrointestinal transit, and gut microbiota in humanized mice. Gastroenterology 144: 967-977, 2013.

18. Samuel BS, Shaito A, Motoike T, Rey FE, Backhed F, Manchester JK, Hammer RE, Williams SC, Crowley J, Yanagisawa $\mathrm{M}$ and Gordon JI: Effect of the gut microbiota on host adiposity are modulated by the short-chain fatty-acid binding G protein-coupled receptor, Gpr41. Proc Natl Acad Sci USA 105: 16767-16772, 2008

19. Sanger GJ: 5-Hydroxytryptamine and functional bowel disorders. Neurogastroenterol Motil 8: 319-331, 1996.

20. Muller PA, Koscsó B, Rajani GM, Stevanovic K, Berres ML, Hashimoto D, Mortha A, Leboeuf M, Li XM, Mucida D, et al: Crosstalk between muscularis macrophages and enteric neurons regulates gastrointestinal motility. Cell 158: 300-313, 2014.

21. Mayer EA, Tillisch K and Gupta A: Gut/brain axis and the microbiota. J Clin Invest 125: 926-938, 2015.

22. Wood JD: Neuropathophysiology of functional gastrointestinal disorders. World J Gastroenterol 13: 1313-1332, 2007.

23. Mawe GM and Hoffman JM: Serotonin signalling in the gut-functions, dysfunctions and therapeutic targets. Nat Rev Gastroenterol Hepatol 10: 473-486, 2013.

24. Gershon MD: Nerves, reflexes, and the enteric nervous system: Pathogenesis of the irritable bowel syndrome. J Clin Gastroenterol 39 (5 Suppl 3): S184-S193, 2005. 